\title{
Realtime Quality Assessment of Iris Biometrics under Visible Light
}

\author{
Mohsen Jenadeleh ${ }^{1,2}$, Marius Pedersen ${ }^{2}$, Dietmar Saupe $^{1}$ \\ ${ }^{1}$ University of Konstanz, Konstanz, Germany \\ ${ }^{2}$ Norwegian University of Science and Technology, Gjøvik, Norway \\ mohsen.jenadeleh@uni-konstanz.de, marius.pedersen@ntnu.no, dietmar.saupe@uni-konstanz.de
}

\begin{abstract}
Ensuring sufficient quality of iris images acquired by handheld imaging devices in visible light poses many challenges to iris recognition systems. Many distortions affect the input iris images, and the source and types of these distortions are unknown in uncontrolled environments. We propose a fast no-reference image quality assessment measure for predicting iris image quality to handle severely degraded iris images. The proposed differential signmagnitude statistics index (DSMI) is based on statistical features of the local difference sign-magnitude transform, which are computed by comparing the local mean with the central pixel of the patch and considering the noticeable variations. The experiments, conducted with a reference iris recognition system and three visible light datasets, showed that the quality of iris images strongly affects the recognition performance. Using the proposed method as a quality filtering step improved the performance of the iris recognition system by rejecting poor quality iris samples.
\end{abstract}

\section{Introduction}

Since the stability of iris patterns over a human lifetime and their uniqueness were noticed in 1987 [11], iris images are used more frequently for identifications and authentications in biometric security applications [7]. Most of the commercially available iris recognition systems use near infrared (NIR) images, but due to the popularity of consumer cameras, iris recognition systems using images acquired under visible light were also developed [37, 49, 39, 40].

Image quality is a key factor that affects the performance of iris recognition systems [48, 47, 3]. There are many distortions that may affect the quality of iris images, including Gaussian blur, motion blur, impulse noise, Gaussian noise, and over-exposure. There are also other quality factors that depend on the content of iris images, including glare, occlusion, and iris deformation. The performance of an iris recognition system under visible light suffers from all of these distortions. To overcome this problem, recently some iris recognition systems have considered the quality of the input iris image [1, 35, 53, 44, 9, 27] in different ways. However, these systems suffer from two major weaknesses:

- The types of distortions considered are limited. Usually only some frequently seen distortions such as Gaussian blur, noise, motion blur, and defocus are considered. However, real-world iris images, especially images taken by handheld devices, may also suffer from other types of distortions simultaneously.

- In related works, usually the quality assessment is applied to accurately segmented iris images, but the quality of iris images also effects the performance of the segmentation. Incorrect iris segmentation increases the false rejection rate.

One of the main goals of this paper is to introduce a general fast image quality assessment method to assess the distortions of the input iris image that can be used to rapidly reject iris samples with poor quality. Also, we investigated the effect of iris image quality on the performance of a reference iris recognition system on three challenging iris image datasets acquired under visible light. Figure 1 shows the general overview of the proposed framework for handheld iris recognition systems under visible light.

The main contributions of this paper are as follows:

1. We introduce a no-reference image quality assessment measure for iris biometrics. First, sign and magnitude patterns are derived. Then, the statistical features of these patterns are analyzed for studying their sensitivity to iris image distortions. Further, statistical features of a specific coincidence sign-magnitude patterns with high sensitivity to image impairment are computed. A weighted nonlinear mapping is applied to the features to form the iris image quality score.

2. We conducted extensive experiments on three visible light datasets from the $G C^{2}$ multi-modal biometric database [25] to analyse the effect of iris image quality filtering on the performance of an iris recognition system. In this study, OSIRIS version 4.1, [32] was 


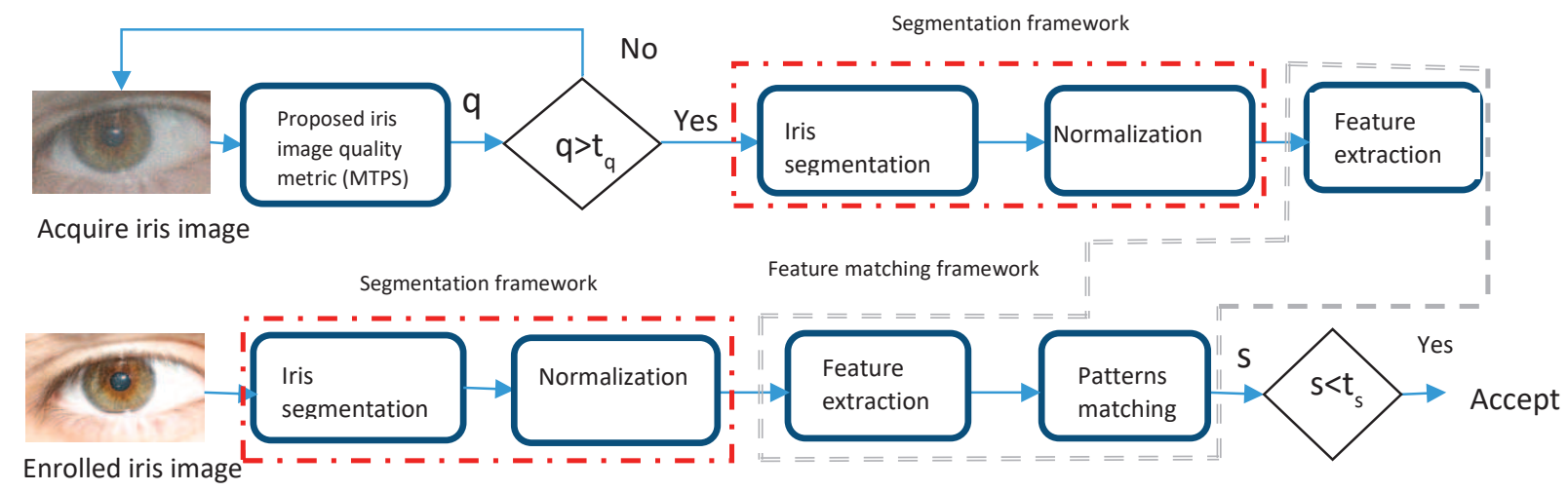

Figure 1. General overview of the suggested framework for handheld iris recognition systems. The proposed image quality measure can be used as a pre-processing step to pass only iris images with sufficient quality to further processing in the iris recognition systems. This approach helps to reduce false negative and false positive authentication due to low image quality. In the figure, $q$ is the image quality score predicted by the proposed DSMI measure, $t_{q}$ is the minimum suitable quality, $s$ is the features matching score, and $t_{s}$ is the threshold of the iris recognition system for accepting or rejecting the pattern matching.

used as a reference iris recognition system. We used three methods to measure the performance improvements: Daugman's decidability index [5], AUC (area under the curve), and EER (equal error rate). The experiments showed the proposed framework provided significant improvements on the performance by rejecting poor quality iris samples.

\section{Related Work}

Recently, research has been reported to improve the performance of iris recognition systems by considering image quality, however with certain limitations. Some iris image quality measures are based on fusion of multiple quality measures. The authors in [18, 20] combined quality measures including motion blur, defocus, off-angle, occlusion, etc. to an overall iris quality score. These methods were designed for NIR-based iris recognition systems and thus, cannot be used directly for iris images under visible light. $\mathrm{Li}$ et al. [21] proposed a method for predicting an iris matching score based on a feature vector of quality measures such as focus, motion blur, illumination, off-angle, occlusions, and dilation. This method needs iris segmentation result for computing quality factors such as dilation and occlusions.

Recently, many machine learning based image quality methods were proposed for quality assessment of natural images [29, 16, 24, 17, 51]. Liu et al. [25] studied some of these methods for filtering low quality iris images. Their experiments showed inconsistencies, i.e., removing more low quality images did not always increase the performance of iris recognition systems.

Belcher et al. [1] measured occlusion and dilation distortions in iris images, and then combined them to a total image quality score to study the correlation between iris images quality and iris recognition accuracy. Their experi- ments showed that image quality could be used for prediction of iris matching score.

Some researches investigated iris image quality considering only specific quality factors such as out-of-focus [50, 45], sharpness [4], and JPEG compression [2]. So they are not generalizable for assessing quality of iris images in presence of a mixture of iris distortions.

In [30], the authors proposed an iris segmentation method, based on the segmentation results, a real time iris quality measure was introduced. This measure cannot be used for estimating iris quality before segmentation.

Happold et al. [14] compute a feature vector of quality related factors, and then labeled the iris images in the training database with their corresponding matching scores. The model can predict the matching scores using the feature vector of certain quality factors. For computing the quality factors the output of the iris segmentation is needed. Therefore, the method cannot be used to measure quality in the iris recognition system pipeline before segmentation.

The fast iris recognition (FIRE) system for images acquired by mobile phones under visible light was proposed by Galdi et al. [12]. It is based on the combination of three classifiers exploiting the iris color and texture information.

A method based on rejecting low quality iris images in visible light was proposed by Proenca [37]. In this method, image degradations such as defocus, motion blur, image angle, iris occlusions, and pupillary dilation are measured from accurately segmented iris patterns. Then, the effect of image quality on feature extraction and matching is considered. The results showed significant performance improvement of the iris recognition system. However, the method has drawbacks. It requires accurately segmented irises, and only a limited number of distortion types are considered.

Recently, OSIRIS version 4.1, an open source iris recog- 
nition system was proposed by Othman et al. [32]. This method follows the Daugman method [6] with improvements in four different modules of OSIRIS, consisting of segmentation, normalization, encoding, and matching. For iris and pupil segmentation, the Viterbi algorithm is used for an optimal contour detection. For normalization a noncircular iris normalization is performed using the coarse contours detected by the Viterbi algorithm. The encoding module is based on 2-D Gabor filters computed at various scales and resolutions. Finally, the matching module computes the global dissimilarity score between two iris codes using Hamming distance. We used this method as a reference iris recognition system.

An efficient deep learning based iris recognition method was proposed by Zhao et al. [52]. The proposed methods includes two sub-networks. MaskNet network for identifying iris regions, and FeatNet for iris feature extraction. For extracting effective iris features by FeatNet, a new Extended Triplet Loss function have been proposed for incorporating a bit-shifting operation into the original loss function for Triplet networks. This method was trained and tested on NIR iris databases.

Some of the iris quality assessment methods such as [18, 20] were proposed for traditional NIR imaging systems and consider a limited distortions. Other methods, such as [37, 14, 30], were introduced for assessing the iris images quality in presence of a limited number of distortion, and they also need the output of the segmentation module of iris recognition systems for generating their quality features. In addition, these systems suffer from a lack of generality required to assess the quality of iris images for unknown distortions in an unconstrained real-world environment.

Iris recognition systems based on images acquired by handheld devices in visible light will broaden the scope of applications of iris recognition and requires more research for suitable fast image quality measures.

\section{Proposed Quality Measure}

In this section, we present a fast and general method for assessing impairment of iris images acquired by handheld devices under visible light.

Local binary patterns (LBP) and their derivatives improved many pattern recognition applications such as texture classification [22, 23, 13], image retrieval [10, 31], object recognition [42, 43], and biometric recognition [33, 19. 26, 46, 36]. Most of the LBP based biometric recognition methods use statistical analysis of the local patterns for their feature extraction. Severities of the image distortions could change these statistics.

We propose an image quality measure to assess the image quality based on the changes on the statistics of a certain coincidence sign-magnitude pattern. The iris image quality measure exploits the observation that low quality iris im- ages have significantly fewer of these patterns compared with those in high quality iris images.

Our quality measure uses statistical features extracted from uniform patterns of a local difference sign-magnitude transform (LDSMT) which decomposes the image into sign and magnitude components [13]. Then the locally weighted statistics of a specific sign-magnitude coincidence patterns are used for formulating the quality score.

These patterns were chosen after statistical analyses of the sign-magnitude patterns of completed local binary patterns [13] representation of an image. In this paper, for simplifying the method, we formulate the selected statistics and do not explain the general procedure of the completed local binary pattern. The patterns were analysed using 4neighborhood with radius 1 of the central pixel of a local patch.

Let $C=\{(i, j) \mid i=0, \ldots, M-1, j=0, \ldots, N-1\}$ be the set of pixels of an image $I$. Replace the grayscale image $I$ by its normalized version $\hat{I}$ :

$$
\hat{I}(c)=\frac{I(c)-I_{\min }}{I_{\max }-I_{\min }}, \quad c \in C
$$

Let the 4-neighborhood of a pixel in position $(0,0)$ be $G=$ $\{(1,0),(0,1),(-1,0),(0,-1)\}$. For all pixels $c \in C$ not on the boundary:

- Compute local maxima and store them in the $S_{1}(c)$ :

$$
S_{1}(c)= \begin{cases}1 & \hat{I}(c)>\max (\hat{I}(c+\epsilon) \mid \epsilon \in G) \\ 0 & \text { otherwise }\end{cases}
$$

- Compute pixels with local differences with respect to threshold $T$ and store them in significance map $S_{2}(c)$ :

$$
S_{2}(c)= \begin{cases}1 & T>\max (\hat{I}(c)-\hat{I}(c+\epsilon) \mid \epsilon \in G) \\ 0 & \text { otherwise }\end{cases}
$$

The threshold $T$ is determined adaptively, here it is the averaged grayscale difference in the entire image.

The statistics of the coincidence pattern of the sign $S_{1}(c)$ and magnitude $S_{2}(c)$ is computed as follows:

$$
S(c)= \begin{cases}1 & S_{1}(c)=1 \text { and } S_{2}(c)=1 \\ 0 & \text { otherwise }\end{cases}
$$

The local variance of the normalized image is:

$$
\sigma^{2}(c)=\operatorname{var} \hat{I}(c+\epsilon) \mid \epsilon \in G
$$

The normalization by the local variance emphasizes the value of the significant local maxima. We define the locally weighted statistics of the coincidence pattern as follows:

$$
Q=r\left(\frac{1}{|C|} \sum_{c \in C} \frac{S(c)}{\sigma^{2}(c)+\delta^{2}}\right)
$$


where $r$ normalizes the scores to $[0,1)$ for ease of interpretation of the quality scores.

$$
\begin{aligned}
r:[0, \infty) & \rightarrow[0,1) \\
x & \mapsto 1-e^{-a x}
\end{aligned}
$$

where $\delta^{2}$ is a small constant to prevent division by zero, and $a$ is a constant for adjusting the nonlinear mapping. $Q=0$ denotes the lowest image quality and $Q \approx 1$ refers to the highest image quality. $Q$ is used as our DSMI iris image quality measure. The parameters $\delta^{2}$ and $a$ were set to be equal to 0.00025 and 0.01 , respectively.

To investigate the discrimination ability of the proposed measure, we generated a synthetically distorted iris image database from 600 high quality reference iris images. These iris images were selected from the WarsawBioBase-Smartphone-Iris v1.0 [49], UTIRIS [15], and $G C^{2}$ databases [25]. 3-12 samples of each eye from 75 individuals were selected as reference iris images. These fully depict the irises, and were selected from individuals with high, medium, and low degrees of iris pigmentations. All reference images were segmented accurately by the reference iris recognition system.

We distorted the reference iris images by five frequently seen distortions with different severities. The distortion types are Gaussian blur, motion blur, Gaussian noise, impulse (salt \& pepper) noise, and over-exposure. We used the following MATLAB functions to generate the distorted versions of a reference image $I$ : Gaussian blur (imgaussfilt(I, sigma)), impulse noise (imnoise $(I$, ' salt\&pepper', density)), overexposure $(\mathrm{I}+\mathrm{c})$, motion blur $\left(H=\right.$ fspecial $\left({ }^{\prime}\right.$ motion', len, theta); imfilter $\left(I, H,{ }^{\prime}\right.$ replicate $\left.{ }^{\prime}\right)$ ), and white Gaussian noise (imnoise $\left(I,{ }^{\prime}\right.$ gaussian', $\left.0, V\right)$ ). The parameters, and the number of distorted versions of each reference image for each distortion type are described briefly in Table 1.

To analyse the discrimination ability of the proposed measure, we plotted in Figure 2 the normalized histogram of the DSMI scores for the reference iris images versus the normalized histogram of the DSMI scores for the distorted iris images for each distortion type separately. Also, the histogram intersection $\left(h_{d}\right)$ between these two histograms is computed for assessment of the discrimination ability of the quality measure. Smaller values indicate better ability for the DSMI scores to discriminate between high quality images and their distorted versions.

\section{Experimental Results}

We investigate whether filtering poor quality iris images improve the performance of an iris recognition system. Most of the existing iris image quality measures need accurately segmented irises for quality assessment [37] and iris image quality could lead to poor iris segmentation. Therefore, in our approach, we reject images in the beginning of the iris recognition pipeline i.e., before segmentation.
Table 1. A brief description of the artificially distorted iris images dataset. In the table, if the kernel has two parameters (e.g. A and B), we showed their intervals using A:B format.

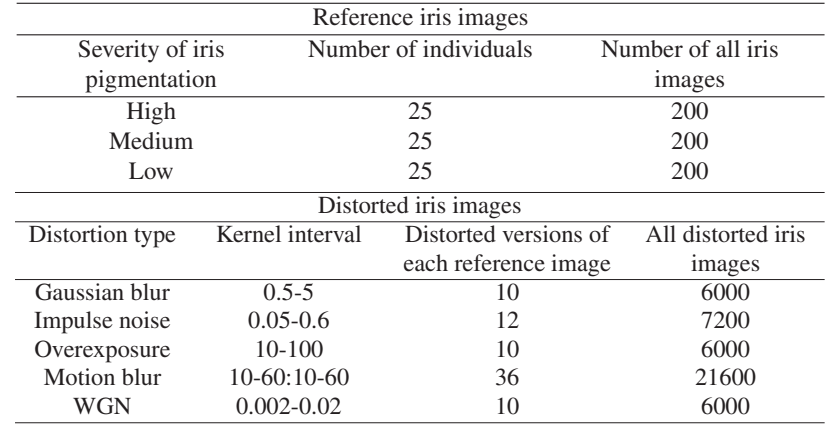

We compare our proposed DSMI measure with two state-of-the-art image quality measures, BRISQUE [28] and WAV1 [34]. BRISQUE uses the statistics of pixel intensities subtracted from local means and normalized by local contrasts to train a regression model for image quality assessment. Pertuz et al. [34] compared 15 methods that can be used for estimating the blurriness of an image. In this study, WAV1 performs best for predicting the blurriness of an image. WAV1 is a quality measure based on statistical properties of the discrete Wavelet transform coefficients.

In our experiments, we illustrate the improvements in the performance of the reference iris recognition system for each image quality measure being used for filtering iris images with poor quality. The experiments were conducted on three iris image datasets acquired under visible light.

\subsection{Databases for Iris Images}

Five iris image databases acquired under visible light are widely used in iris recognition research: UTIRIS [15], UBIRIS [38], MICHE [8], and VISOB [41]. The UTIRIS iris images were acquired with an optometric framework and in a controlled environment that resulted in high quality images.

UBIRIS iris images were acquired from moving subjects and varying distances which resulted in more heterogeneous images compared with UTIRIS. Still the images have a good quality, better than typically attained by smartphones in uncontrolled environments. The MICHE and VISOB databases are challenging databases for iris recognition systems including images with different degrees of iris pigmentation and with eye makeup. Moreover, the quality of the images is affected by lacking focus, occlusions due to prescription glasses, different illumination conditions, gaze deviations, specular reflections, and motion blur.

Instead of the above, we chose the multi-modal biometric database $G C^{2}$ [25] because it includes more authentically distorted iris images that typically may occur when users capture iris images with their smartphones in uncontrolled environments. Also, the number of images per eye 


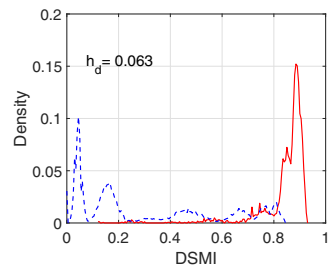

(a) Gaussian blur

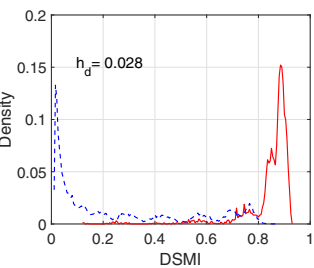

(b) Impulse noise

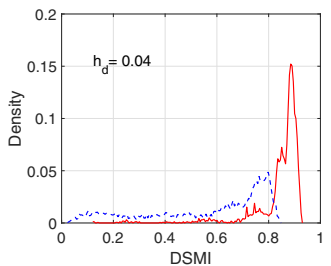

(c) Overexposure

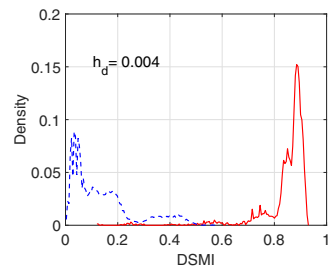

(d) Motion blur

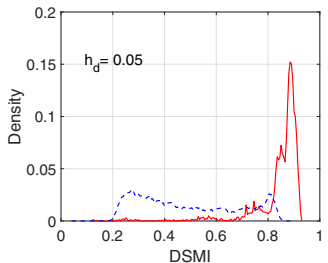

(e) WGN

Figure 2. Red solid lines show the normalized histogram of the DSMI quality scores for high quality iris images, and the dotted blue lines show the histograms for the distorted versions with different distortion types. $h_{d}$ is the histogram intersection value.

\begin{tabular}{lccc}
\multicolumn{4}{c}{ Table 2. Summary of $G C^{2}$ database } \\
\hline Datasets & REFLEX & LFC & PHONE \\
\hline \hline Number of Subjects & 48 & 49 & 50 \\
Total images & 1422 & 1454 & 1379 \\
samples per eye & $12-15$ & $13-15$ & $12-15$ \\
Matching pairs & 9457 & 10045 & 9092 \\
Non-matching pairs & 975450 & 1056485 & 941039 \\
Camera & Canon D700 & Light field camera & Phone nexus \\
\hline
\end{tabular}

in $C G^{2}$ is larger than in the other databases and the images show a wider range of iris image impairment.

We used three datasets from $G C^{2}$. The iris images in these three datasets were captured in different lighting conditions and with different cameras in uncontrolled environments at varying distances and from subjects with different degrees of iris pigmentation. The first dataset, REFLEX, was captured using a Canon D700 camera with Canon EF $100 \mathrm{~mm} \mathrm{f} / 2.8 \mathrm{~L}$ macro lens (18 megapixels). It contains 1422 iris images from 48 subjects. 12 to 15 samples were taken per eye (left and right). The second dataset, LFC, contains iris images captured by a light field camera. It contains 1454 iris images of the left and right eyes of 49 subjects, and 13 to 15 samples were taken per eye. The third dataset, PHONE, was captured by a smartphone (Google Nexus 5, 8 megapixels) and contains 1379 iris images of both eyes of 50 subjects. 12 to 15 samples were taken per eye.

We compare one iris image against all iris images from the same dataset. Table 2 summarizes these datasets and shows the number of matching and non-matching pairs.

\subsection{Iris Recognition Performance Analysis}

To evaluate the performance improvement in the iris recognition system achieved due to iris quality filtering using an image quality metric, we used three methods: the Daugman's decidability index [5], the area under the receiver operating characteristic curves (AUC), and the equal error rates (EER). Three thresholds for the respective metric were chosen so that $1 / 4,1 / 2,3 / 4$ of the iris images with lowest quality were rejected. In thiy way, we compared the performance of our proposed DSMI metric with BRISQUE and WAV1. In our experiments, the reference iris recognition system, OSIRIS, version 4.1, was used.

\subsubsection{Daugman's Decidability Index}

Daugman's decidability index [5] is widely used for assessing the performance of iris recognition systems [5, 37, 25]. The index $\left(d^{\prime}\right)$ measures separation of the distribution of the matching iris scores from the distribution of the nonmatching iris scores:

$$
d^{\prime}=\frac{\left|\mu_{E}-\mu_{I}\right|}{\sqrt{\frac{1}{2}\left(\sigma_{I}^{2}+\sigma_{E}^{2}\right)}}
$$

where $\mu_{E}$ and $\mu_{I}$ are the means and $\sigma_{E}$ and $\sigma_{I}$ are the standard deviations of the distributions of scores of the matching and the non-matching iris pairs. Larger values correspond to better discrimination in iris recognition systems.

In OSIRIS, version 4.1, the fractional Hamming distance of the feature vectors gives the dissimilarity score between pair of iris images. We plot the matching and the nonmatching normalized histograms of the Hamming distances of iris pairs. For visualization, normal distributions were fitted to the histograms (Figure 3).

Rejecting samples with minimum quality moves the distributions of Hamming distances for the matching irises to the left, but has little effect on the distribution for the nonmatching iris distances (Figure 3 (a)). This right-shift of the matching distribution increases the decidability index and improves the iris recognition performance. In the remaining parts of the figure, we removed the distributions of the nonmatching scores and plotted only the normal distributions fitted to the histograms of the matching scores for clarity. Also, the corresponding Daugman's decidability index $\left(d^{\prime}\right)$ values are shown in the figure.

In Figure 3 (c) BRISQUE was used for quality filtering, and in Figure 3 (d) WAV1 was used for rejecting the images with poorest quality. As can be seen in the figures, filtering the poor quality images using DSMI and BRISQUE improve the performance of the reference iris recognition system in the REFLEX dataset, but WAV1 deteriorates the performance. We performed the same experiments for the LFC and PHONE datasets using three image quality measures for rejecting iris image with minimum quality.

From the decidability index values in the three testing datasets as shown in Figure 3, we can conclude that filtering 

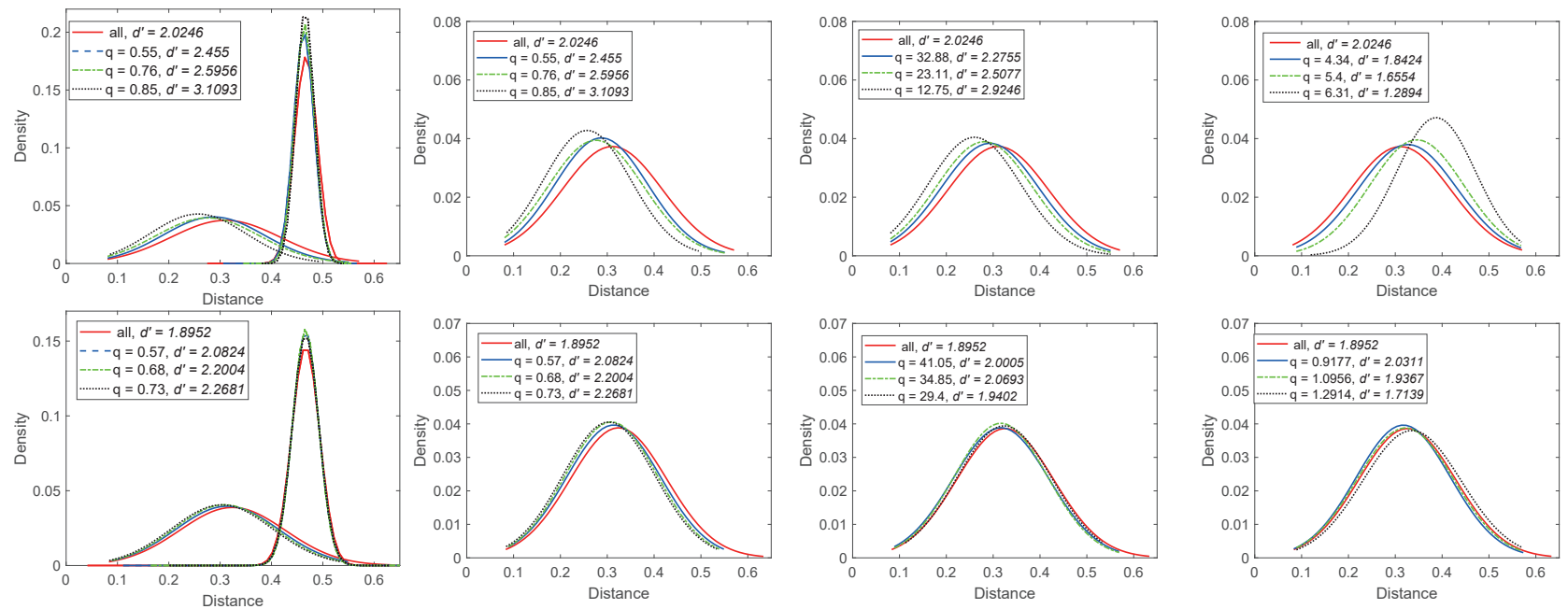

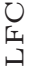

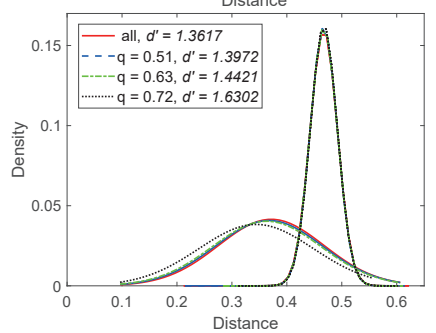

(a) DSMI

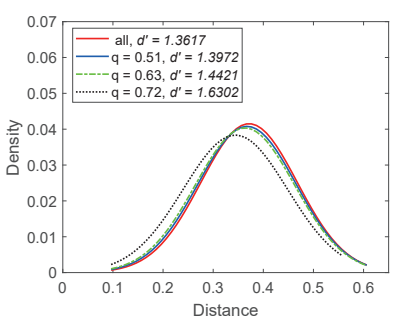

(b) DSMI

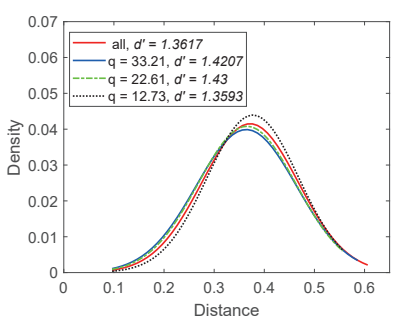

(c) BRISQUE

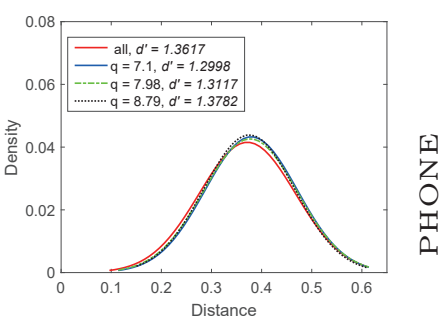

(d) WAV1

Figure 3. The normal distributions fitted on the normalized histograms of the fractional Hamming distances for the matching irises, and non-matching irises according to iris images quality filtering are illustrated for the REFLEX dataset. Also, the Daugman decidability index $\left(d^{\prime}\right)$ value corresponding to each quality threshold is computed. Because the non-matching distributions do not change with quality filtering, only the matching score distributions are illustrated for better visualization. For DSMI and WAV1, a higher score indicates a better quality, where for BRISQUE a lower score indicates better quality.

the iris images with poor quality using the proposed DSMI measure, improves the recognition accuracy of the reference iris recognition system. The BRISQUE measure performs well in the REFLEX dataset, but it is not consistent for quality filtering in LFC and PHONE datasets. WAV1 is not consistent on all the testing datasets.

\subsubsection{Receiver Operating Characteristic Curve}

The area under the curve (AUC) of the receiver operating characteristic (ROC) is widely used for comparing the accuracy of iris recognition systems. With this approach, the system with the larger AUC is considered more accurate.

For visualizing and measuring the improvements on the performance of the reference iris recognition system due to quality filtering of iris images, we created ROC curves for each dataset by plotting the true acceptance rate against the false acceptance rate at various fractional Hamming distance threshold settings (Figure 44). We computed the AUC values, listed in the figure legends.

Without quality filtering the AUC value (red solid lines) for REFLEX dataset is $0.9061,0.8861$ for LFC, and 0.8226 for PHONE. This shows that the PHONE dataset is the most challenging one for the reference iris recognition system. Figure 4 also shows how the AUC values change with different iris image quality filtering thresholds on the three test datasets using the three different image quality measures.

From the results, we conclude that the performance of the reference iris recognition system improved increasingly by rejecting more and more iris images with poorest quality, when using the DSMI measure. In contrast, BRISQUE is consistent for quality assessment only for the REFLEX dataset, but not on the other two datasets. WAV1 shows inconsistent performance in all test datasets.

\subsubsection{Equal Error Rate Measure}

The equal error rate (EER) is the rate at which both accept and reject errors are equal. EER is used for comparing the accuracy of classification systems with different ROC curves. With the EER approach, the system with the lowest EER is considered the most accurate.

Figure 5 shows the false positive rates versus the false negative rates for iris pairs for the REFLEX, LFC, and 

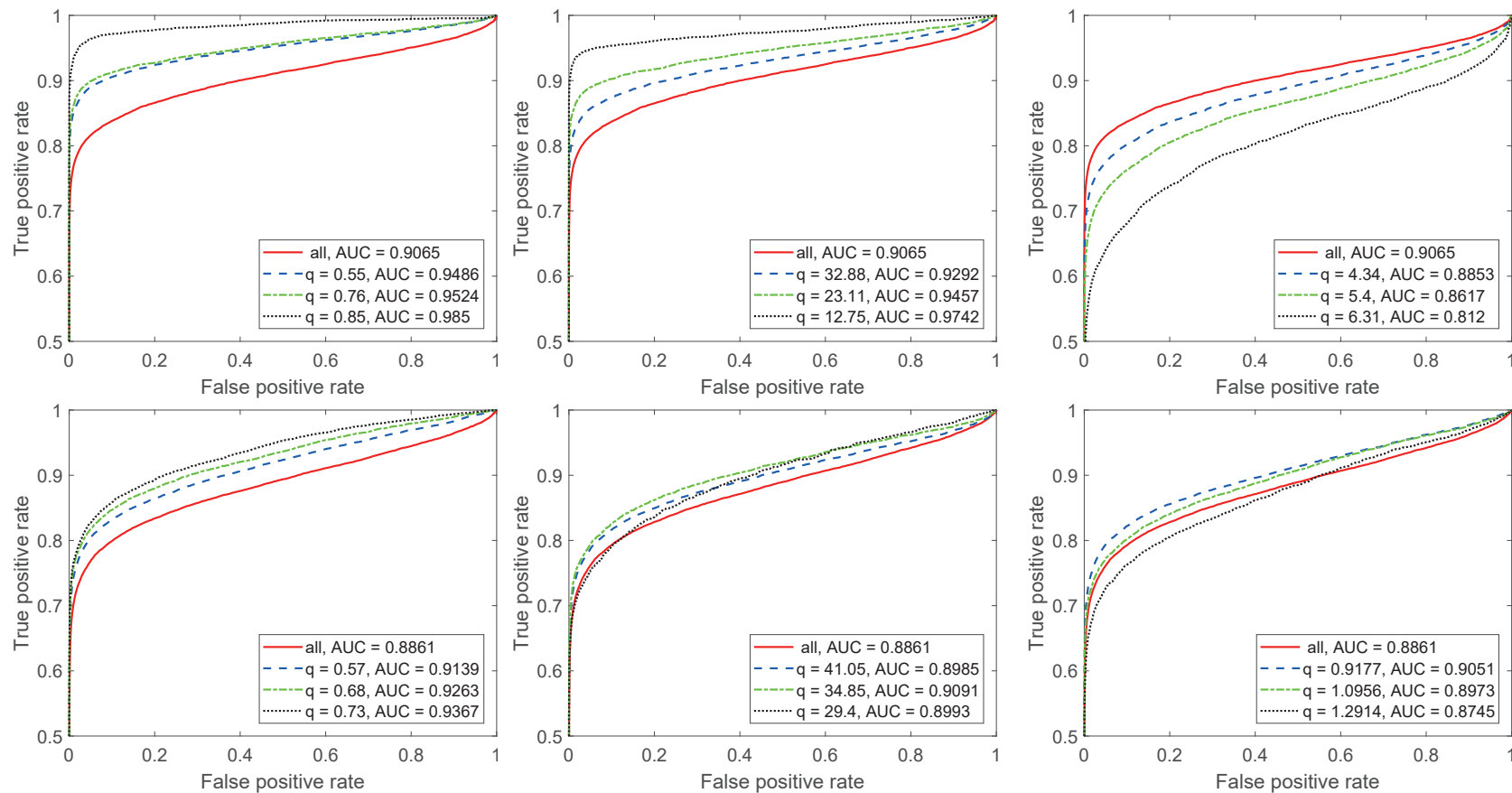

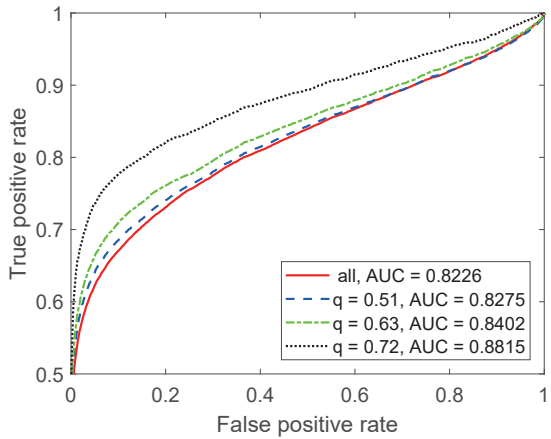

(a) DSMI

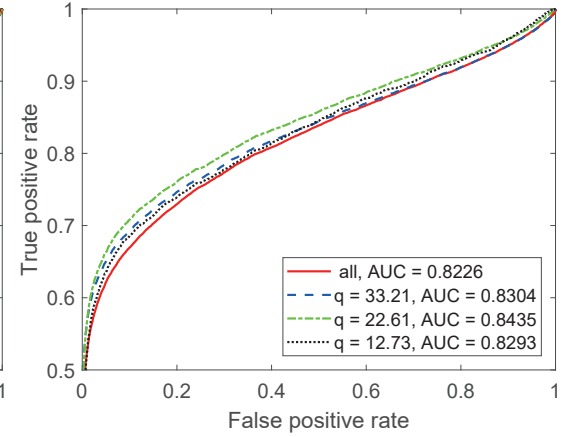

(b) BRISQUE

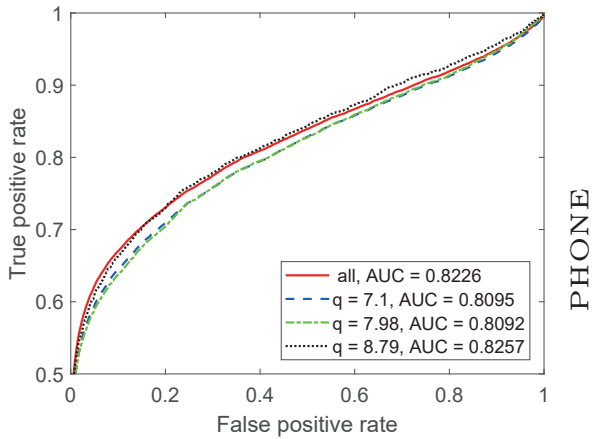

(c) WAV1

Figure 4. The area under the curve (ROC) curves corresponding to the iris images quality filtering. Red solid line, blue dashed line, dotdashed green line, and the dotted black line were plotted for all images, after filtering a quarter, half, and three quarters of the iris images with minimum quality respectively. We performed the quality filtering using (a) DSMI, (b) BRISQUE, and (c) WAV1.

PHONE datasets. We computed the EER values, listed in the figure legends and shown as bullet points on the curves.

The results confirm that rejecting poor quality images using DSMI improves iris recognition performance consistently, while this does not hold for BRISQUE and WAV1.

In summary, for all of the test sets (REFLEX, LFC, PHONE) and all of the evaluation methods (Daugman's decidability index, AUC, EER) the performance of the reference iris recognition system (OSIRIS, Version 4.1) increased consistently by filtering iris images with minimum quality using the proposed DSMI quality metric. In contrast, for the other two image quality metrics (BRISQUE, WAV1), the experiments showed inconsistencies, i.e., removing more low quality images did not always increase performance.

\subsection{Computational Complexity}

The computational complexity of the proposed DSMI measure can be roughly estimated using its run time. For this aim, we assessed the quality of four sets of iris images with different resolutions from the testing datasets using the proposed DSMI measure.

We used a T430 Lenovo laptop with an Intel Core i5 processor and 6GB RAM with MATLAB version 2017a in Ubuntu 16.04.3 LTS to run the DSMI metric, and the average run time and frames per second for each set with different resolutions are reported in Table 3 .

The results show that the proposed method can be used to assess the quality iris images in interactive applications such as handheld based iris recognition systems. 

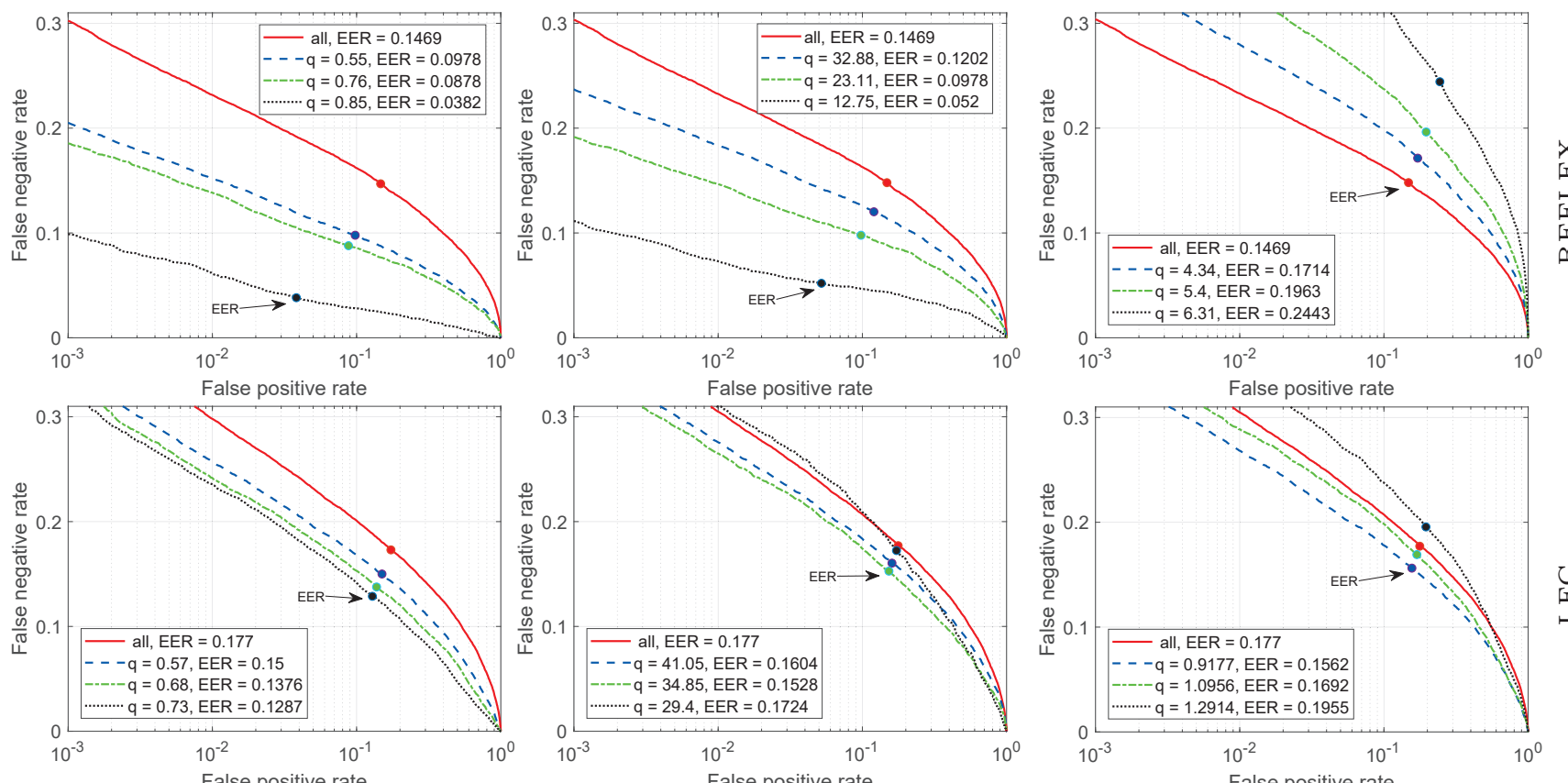

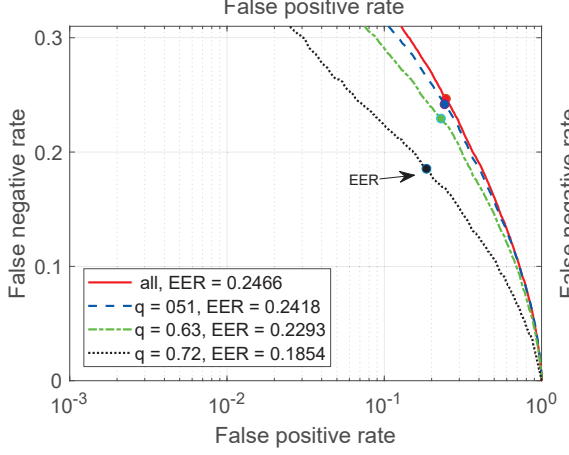

(a) DSMI

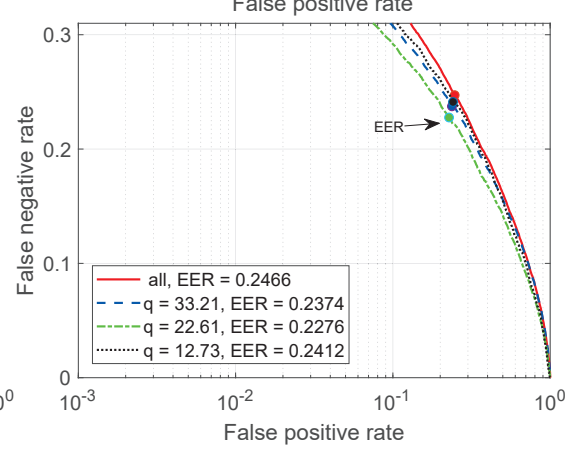

(b) BRISQUE

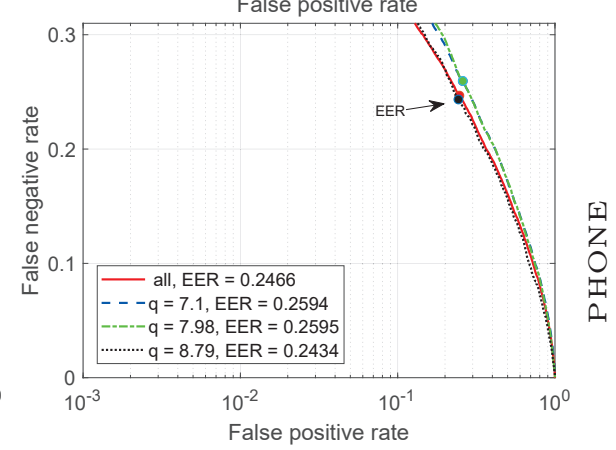

(c) WAV1

Figure 5. The equal error rate (EER) curves corresponding to the iris images quality filtering. Red sold line, blue dashed line, dot-dashed green line, and the dotted black line were plotted for all images, after filtering a quarter, half, and three quarters of the iris images with minimum quality respectively. We performed the quality filtering using (a) DSMI, (b) BRISQUE, and (c) WAV1 quality measures.

Table 3. Average run time (seconds) on four sets of iris images.

\begin{tabular}{lcccc}
\hline Image resolutions & $453 \times 303$ & $625 \times 417$ & $822 \times 548$ & $1352 \times 920$ \\
Average run time & 0.015 & 0.024 & 0.041 & 0.106 \\
Frames per second & 66 & 40 & 24 & 9 \\
\hline
\end{tabular}

\section{Conclusions and Future Work}

In this paper, we presented a new training free, general, and realtime image quality measure, based on statistical features of the sign-magnitude transform to estimate the quality of iris images acquired by handheld devices under visible light.

We suggest that this method can be used for rejecting poor quality iris images from the iris recognition pipeline to improve the recognition rate of the reference iris recognition system. Experiments showed that the proposed approach improved the accuracy of a reference iris recognition system.
We remark, however, that the inclusion of the quality filtering step in an iris recognition system (see Figure 1), may increase the computational cost; and some iris images may be rejected unnecessarily. This could be caused by a failure of the quality measure or by a setting of the quality threshold that is too conservative.

In our future work, we will propose a performance measure for iris recognition systems that considers all these factors together.

\section{Acknowledgment}

We thank the German Research Foundation (DFG) for financial support within project A05 of SFB/Transregio 161. Our work was partially funded by the Research Council of Norway through project number 221073: HyPerCept Color and Quality in Higher Dimensions. 


\section{References}

[1] C. Belcher and Y. Du. A selective feature information approach for iris image-quality measure. IEEE Transactions on Information Forensics and Security, 3(3):572-577, 2008.

[2] T. Bergmüller, E. Christopoulos, K. Fehrenbach, M. Schnöll, and A. Uhl. Recompression effects in iris recognition. Image and Vision Computing, 58:142-157, 2017.

[3] S. Bharadwaj, M. Vatsa, and R. Singh. Biometric quality: a review of fingerprint, iris, and face. EURASIP Journal on Image and Video Processing, 2014(1):34, 2014.

[4] L. Chen, M. Han, and H. Wan. The fast iris image clarity evaluation based on brenner. In Instrumentation and Measurement, Sensor Network and Automation (IMSNA), 2013 2nd International Symposium on, pages 300-302. IEEE, 2013.

[5] J. Daugman. Biometric decision landscapes. Technical Report 482, University of Cambridge, Computer Laboratory, 2000. Available at.

[6] J. Daugman. How iris recognition works. IEEE Transactions on circuits and systems for video technology, 14(1):21-30, 2004.

[7] J. Daugman. New methods in iris recognition. IEEE Transactions on Systems, Man, and Cybernetics, Part B (Cybernetics), 37(5):1167-1175, 2007.

[8] M. De Marsico, M. Nappi, D. Riccio, and H. Wechsler. Mobile iris challenge evaluation (miche)-i, biometric iris dataset and protocols. Pattern Recognition Letters, 57:17-23, 2015.

[9] W. Dong, Z. Sun, T. Tan, and Z. Wei. Quality-based dynamic threshold for iris matching. In Image Processing (ICIP), 2009 16th IEEE International Conference on, pages 19491952. IEEE, 2009.

[10] S. R. Dubey, S. K. Singh, and R. K. Singh. Multichannel decoded local binary patterns for content-based image retrieval. IEEE Transactions on Image Processing, 25(9):4018-4032, 2016.

[11] L. Flom and A. Safir. Iris recognition system, Feb. 31987. US Patent 4,641,349.

[12] C. Galdi and J.-L. Dugelay. Fire: Fast iris recognition on mobile phones by combining colour and texture features. Pattern Recognition Letters, 2017.

[13] Z. Guo, L. Zhang, and D. Zhang. A completed modeling of local binary pattern operator for texture classification. IEEE Transactions on Image Processing, 19(6):1657-1663, 2010.

[14] M. Happold. Learning to predict match scores for iris image quality assessment. In Biometrics (IJCB), 2014 IEEE International Joint Conference on, pages 1-8. IEEE, 2014.

[15] M. S. Hosseini, B. N. Araabi, and H. Soltanian-Zadeh. Pigment melanin: Pattern for iris recognition. IEEE Transactions on Instrumentation and Measurement, 59(4):792-804, 2010.

[16] M. Jenadeleh, M. M. Masaeli, and M. E. Moghaddam. Blind image quality assessment based on aesthetic and statistical quality-aware features. Journal of Electronic Imaging, 26(4):043018, 2017.

[17] M. Jenadeleh and M. E. Moghaddam. Biqws: efficient wakeby modeling of natural scene statistics for blind im- age quality assessment. Multimedia Tools and Applications, 76(12):13859-13880, 2017.

[18] N. D. Kalka, J. Zuo, N. A. Schmid, and B. Cukic. Estimating and fusing quality factors for iris biometric images. IEEE Transactions on Systems, Man, and Cybernetics-Part A: Systems and Humans, 40(3):509-524, 2010.

[19] W. Kang and Q. Wu. Contactless palm vein recognition using a mutual foreground-based local binary pattern. IEEE transactions on Information Forensics and Security, 9(11):19741985, 2014.

[20] X. Li, Z. Sun, and T. Tan. Comprehensive assessment of iris image quality. In Image Processing (ICIP), 2011 18th IEEE International Conference on, pages 3117-3120. IEEE, 2011.

[21] X. Li, Z. Sun, and T. Tan. Predict and improve iris recognition performance based on pairwise image quality assessment. In Biometrics (ICB), 2013 International Conference on, pages 1-6. IEEE, 2013.

[22] L. Liu, P. Fieguth, Y. Guo, X. Wang, and M. Pietikäinen. Local binary features for texture classification: Taxonomy and experimental study. Pattern Recognition, 62:135-160, 2017.

[23] L. Liu, S. Lao, P. W. Fieguth, Y. Guo, X. Wang, and M. Pietikäinen. Median robust extended local binary pattern for texture classification. IEEE Transactions on Image Processing, 25(3):1368-1381, 2016.

[24] L. Liu, B. Liu, H. Huang, and A. C. Bovik. Noreference image quality assessment based on spatial and spectral entropies. Signal Processing: Image Communication, 29(8):856-863, 2014.

[25] X. Liu, M. Pedersen, C. Charrier, and P. Bours. Can noreference image quality metrics assess visible wavelength iris sample quality? In IEEE International Conference on Image Processing, pages 3530-3534, 2017.

[26] W. Louis, M. Komeili, and D. Hatzinakos. Continuous authentication using one-dimensional multi-resolution local binary patterns (1dmrlbp) in ecg biometrics. IEEE Transactions on Information Forensics and Security, 11(12):28182832, 2016.

[27] S. Makinana, J. J. Van Der Merwe, and T. Malumedzha. A fourier transform quality measure for iris images. In Biometrics and Security Technologies (ISBAST), 2014 International Symposium on, pages 51-56. IEEE, 2014.

[28] A. Mittal, A. K. Moorthy, and A. C. Bovik. No-reference image quality assessment in the spatial domain. IEEE Transactions on Image Processing, 21(12):4695-4708, 2012.

[29] A. K. Moorthy and A. C. Bovik. A two-step framework for constructing blind image quality indices. IEEE Signal processing letters, 17(5):513-516, 2010.

[30] M. Mottalli, M. Mejail, and J. Jacobo-Berlles. Flexible image segmentation and quality assessment for real-time iris recognition. In Image Processing (ICIP), 2009 16th IEEE International Conference on, pages 1941-1944. IEEE, 2009.

[31] S. Murala and Q. J. Wu. Local mesh patterns versus local binary patterns: biomedical image indexing and retrieval. IEEE Journal of Biomedical and Health Informatics, 18(3):929-938, 2014. 
[32] N. Othman, B. Dorizzi, and S. Garcia-Salicetti. Osiris: An open source iris recognition software. Pattern Recognition Letters, 82:124-131, 2016.

[33] C. N. Padole and H. Proenca. Periocular recognition: Analysis of performance degradation factors. In Biometrics (ICB), 2012 5th IAPR International Conference on, pages 439-445. IEEE, 2012.

[34] S. Pertuz, D. Puig, and M. A. Garcia. Analysis of focus measure operators for shape-from-focus. Pattern Recognition, 46(5):1415-1432, 2013.

[35] J. K. Pillai, V. M. Patel, R. Chellappa, and N. K. Ratha. Secure and robust iris recognition using random projections and sparse representations. IEEE transactions on pattern analysis and machine intelligence, 33(9):1877-1893, 2011.

[36] K. Popplewell, K. Roy, F. Ahmad, and J. Shelton. Multispectral iris recognition utilizing hough transform and modified lbp. In Systems, Man and Cybernetics (SMC), 2014 IEEE International Conference on, pages 1396-1399. IEEE, 2014.

[37] H. Proença. Quality assessment of degraded iris images acquired in the visible wavelength. IEEE Transactions on Information Forensics and Security, 6(1):82-95, 2011.

[38] H. Proenca, S. Filipe, R. Santos, J. Oliveira, and L. A. Alexandre. The ubiris. v2: A database of visible wavelength iris images captured on-the-move and at-a-distance. IEEE Transactions on Pattern Analysis and Machine Intelligence, 32(8):1529, 2010.

[39] K. B. Raja, R. Raghavendra, and C. Busch. Binarized statistical features for improved iris and periocular recognition in visible spectrum. In Biometrics and Forensics (IWBF), 2014 International Workshop on, pages 1-6. IEEE, 2014.

[40] K. B. Raja, R. Raghavendra, V. K. Vemuri, and C. Busch. Smartphone based visible iris recognition using deep sparse filtering. Pattern Recognition Letters, 57:33-42, 2015.

[41] A. Rattani, R. Derakhshani, S. K. Saripalle, and V. Gottemukkula. Icip 2016 competition on mobile ocular biometric recognition. In Image Processing (ICIP), 2016 IEEE International Conference on, pages 320-324. IEEE, 2016.

[42] A. Satpathy, X. Jiang, and H.-L. Eng. Lbp-based edgetexture features for object recognition. IEEE Transactions on Image Processing, 23(5):1953-1964, 2014.

[43] J. Shang, C. Chen, X. Pei, H. Liang, H. Tang, and M. Sarem. A novel local derivative quantized binary pattern for object recognition. The Visual Computer, 33(2):221-233, 2017.

[44] C. Shi and L. Jin. A fast and efficient multiple step algorithm of iris image quality assessment. In Future Computer and Communication (ICFCC), 2010 2nd International Conference on, volume 2, pages V2-589. IEEE, 2010.

[45] V. Starovoitov, A. K. Golińska, A. Predko-Maliszewska, and M. Goliński. No-reference image quality assessment for iris biometrics. In Image Processing and Communications Challenges 4, pages 95-100. Springer, 2013.

[46] A. Suruliandi, K. Meena, and R. R. Rose. Local binary pattern and its derivatives for face recognition. IET computer vision, 6(5):480-488, 2012.

[47] s. Thavalengal, P. Bigioi, and P. Corcoran. Evaluation of combined visible/NIR camera for iris authentication on smartphones. In Proceedings of the IEEE Conference on
Computer Vision and Pattern Recognition Workshops, pages 42-49, June 2015.

[48] S. Thavalengal, P. Bigioi, and P. Corcoran. Iris authentication in handheld devices-considerations for constraint-free acquisition. IEEE Transactions on Consumer Electronics, 61(2):245-253, 2015.

[49] M. Trokielewicz. Iris recognition with a database of iris images obtained in visible light using smartphone camera. In Identity, Security and Behavior Analysis (ISBA), 2016 IEEE International Conference on, pages 1-6. IEEE, 2016.

[50] J. Wan, X. He, and P. Shi. An iris image quality assessment method based on laplacian of gaussian operation. In MVA, pages 248-251. Citeseer, 2007.

[51] L. Zhang, L. Zhang, and A. C. Bovik. A feature-enriched completely blind image quality evaluator. IEEE Transactions on Image Processing, 24(8):2579-2591, 2015.

[52] Z. Zhao and A. Kumar. Towards more accurate iris recognition using deeply learned spatially corresponding features. In Proceedings of the IEEE International Conference on Computer Vision, Venice, Italy, pages 22-29, 2017.

[53] Z. Zhou, E. Y. Du, C. Belcher, N. L. Thomas, and E. J. Delp. Quality fusion based multimodal eye recognition. In Systems, Man, and Cybernetics (SMC), 2012 IEEE International Conference on, pages 1297-1302. IEEE, 2012. 